\title{
A Note on the Paper "Generalized $\phi$-Contraction for a Pair of Mappings on Cone Metric Spaces”
}

\author{
Mohamed Abd El-Rahman Ahmed \\ Department of Mathematics, Faculty of Science, Assiut University, Assiut, Egypt \\ Email: mahmed68@yahoo.com
}

Received August 10, 2011; revised September 20, 2011; accepted September 28, 2011

\begin{abstract}
We note that Theorem 2.3 [1] is a consequence of the same theorem for one map.
\end{abstract}

Keywords: Generalized $\phi$-Contraction; Weakly Compatible; Solid Cone

\section{Introduction}

Huang and Zhang [2] initiated fixed point theory in cone metric spaces. On the other hand, the authors [3] gave a lemma and showed that some fixed point generalizations are not real generalizations. In this note, we show that Theorem 2.3 [1] is so.

Following [2], let $E$ be a real Banach space and $\theta$ be the zero vector in $E$, and $P \subseteq E$. $P$ is called cone iff

1) $P$ is closed, nonempty and $P \neq\{\theta\}$,

2) $a x+b y \in P$ for all $x, y \in P$ and nonnegative real numbers $a, b$,

3) $P \cap(-P)=\{\theta\}$.

For a given cone $P$, we define a partial ordering $\preceq$ with respect to $P$ by $x \preceq y$ iff $y-x \in P$. $x \prec y \quad$ (resp. $x \ll y$ ) stands for $x \preceq y$ and $x \neq y$ (resp. $y-x \in$ $\operatorname{int}(P))$, where int $(P)$ denotes the interior of $P$. In the paper we always assume that $P$ is solid, i.e., $\operatorname{int}(P) \neq \phi$. It is clear that $x \ll y$ leads to $x \preceq y$ but the reverse need not to be true.

The cone $P$ is called normal if there exists a number $K>0$ such that for all $x, y \in E, \theta \preceq x \preceq y$ implies $\|x\| \leq K\|y\|$.

The least positive number satisfying above is called the normal constant of $P$.

Definition 1.1 [2]. Let $X$ be a nonempty set. A function $d: X \times X \rightarrow E$ is called cone metric iff

$$
\begin{aligned}
& \left(M_{1}\right) \quad \theta \preceq d(x, y), \\
& \left(M_{2}\right) \quad d(x, y)=d(y, x)=\theta \text { iff } x=y, \\
& \left(M_{3}\right) \quad d(x, y)=d(y, x), \\
& \left(M_{4}\right) \quad d(x, y) \preceq d(x, z)+d(z, y),
\end{aligned}
$$

for all $x, y, z \in X,(X, d)$ is said to be a cone metric space.

In [3], the authors gave the following important lemma.

Lemma 1.1 Let $X$ be a nonempty and $f: X \rightarrow X$. Then there exists a subset $Y \subseteq X$ such that $f(Y)=$ $f(X)$ and $f: Y \rightarrow X$ is one-to-one.

Definition 1.2 [4]. Let $(X, d)$ be a cone metric space and $f, g: X \rightarrow X$ be mappings. Then, $z \in X$ is called a coincidence point of $f$ and $g$ iff $f(z)=g(z)$.

Definition 1.3 [1]. Let $(X, d)$ be a cone metric space. The mappings $f, g: X \rightarrow X$ are weakly compatible iff for every coincidence point $z \in X$ of $f$ and $g$, $f(g(x))=g(f(x))$.

Definition 1.4 (see [1]). Let $P$ be a solid cone in a real Banach space $E$. A nondecreasing function $\phi: P \rightarrow P$ is called a comparison function iff

1) $\phi(\theta)=\theta$ and $\theta \prec \phi(x) \prec x$ for $x \in P-\{\theta\}$;

2) $x \in \operatorname{int}(P)$ implies $x-\phi(x) \in \operatorname{int}(P)$;

3) $\lim _{n \rightarrow \infty} \phi^{n}(x)=0$ for all $x \in P-\{\theta\}$.

In [1], the authors established the following fixed point theorem.

Theorem 1.1 Let $(X, d)$ be a cone metric space, $P$ a solid cone and $f, g: X \rightarrow X$. Assume that $(f, g)$ is a generalized $\phi$-contraction; i.e., $d(f(x), f(y)) \preceq \varphi(u)$ for all $x, y \in X$ and some $u$ where

$$
\begin{aligned}
u \in\{d(g(x), g(y)), d(f(x), g(x)), \\
\left.\quad d(f(y), g(y)), \frac{d(g(x), f(y))+d(g(y), f(x))}{2}\right\} .
\end{aligned}
$$

Suppose that $f(X) \subseteq g(X), f(X)$ or $g(X)$ is a complete subspace of $X$, and $f$ and $g$ are weakly compati- 
ble. Then the mappings $f$ and $g$ have a unique common fixed point in $X$.

\section{Main Result}

In Theorem 1.1, if we choose $g=I_{X} \quad\left(I_{X}:=\right.$ the identity map on $X$ ), then we have the following theorem.

Theorem 2.1 Let $(X, d)$ be a cone metric space, $P$ a solid cone and $f: X \rightarrow X$. Assume that $f$ is a generalized $\phi$-contraction; i.e., $d(f(x), f(y)) \preceq \phi(u)$ for all $x, y \in X$ and some $u$ where

$$
\begin{gathered}
u \in\{d(x, y), d(x, f(x)), d(y, f(y)), \\
\left.\frac{d(x, f(x))+d(y, f(y))}{2}\right\} .
\end{gathered}
$$

Suppose that $f(X)$ or $X$ is a complete subspace of $X$. Then the mapping $f$ has a unique fixed point in $X$.

Now, we state and prove our main result in the following way.

Theorem 2.2 Theorem 1.1 is a consequence of Theorem 2.1.

Proof. By Lemma 1.1, there exists $Y \subseteq X$ such that $g(Y)=g(X)$ and $g: Y \rightarrow X$ is one-to-one. Define a map $h: g(Y) \rightarrow g(Y)$ by $h(g(x))=f(x)$ for each $x \in g(Y)$. Since $g$ is one-to-one on $Y$, then $h$ is welldefined. $d(f(x), f(y)) \preceq \phi(u)$ for all $x, y \in X$ and some $u$ where

$$
\begin{aligned}
& u \in\{d(g(x), g(y)), d(f(x), g(x)) \\
&\left.d(f(y), g(y)), \frac{d(g(x), f(y))+d(g(y), f(x))}{2}\right\} .
\end{aligned}
$$

Since $f(X)$ or $g(Y)=g(X)$ is complete, by using Theorem 2.1, there exists $x_{0} \in X$ such that $h\left(g\left(x_{0}\right)\right)=g\left(x_{0}\right)=f\left(x_{0}\right)$. Hence, $f$ and $g$ have a point of coincidence which is also unique. Since $f$ and $g$ are weakly compatible, then $f$ and $g$ have a unique common fixed point.

Remark 2.1 Since Theorem 1 [4] is a special case of Theorem 1.1, then it is a consequence of Theorem 2.1, too.

\section{REFERENCES}

[1] A. Razani, V. Rakocevic and Z. Goodarzi, "Generalized $\phi$-Contraction for a Pair of Mappings on Cone Metric Spaces,” Applied Mathematics and Computation, Vol. 217, No. 22, 2011, pp. 8899-8906.

[2] L.-G. Huang and X. Zhang, "Cone Metric Spaces and Fixed Point Theorems of Contractive Mappings,” Journal of Mathematical Analysis and Applications, Vol. 332, No. 2, 2007, pp. 1468-1476. doi:10.1016/j.jmaa.2005.03.087

[3] R. H. Haghi, Sh. Rezapour and N. Shahzad, "Some Fixed Point Generalizations Are Not Real Generalizations," Nonlinear Analysis, Vol. 74, 2011, pp. 1799-1803.

[4] C. D. Bari and P. Vetro, “ $\phi$-Pairs and Common Fixed Points in Cone Metric Spaces,” Rendiconti del Circolo Matematico di Palermo, Vol. 57, No. 2, 2008, pp. 279285. doi:10.1007/s12215-008-0020-9 